\title{
A HER2 bispecific antibody can be efficiently expressed in Escherichia coli with potent cytotoxicity
}

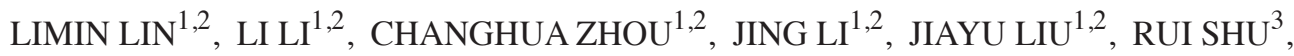 \\ $\mathrm{BIN} \mathrm{DONG}^{4}$, QING LI ${ }^{1,2}$ and ZHONG WANG ${ }^{1,2}$ \\ ${ }^{1}$ School of Pharmaceutical Sciences; ${ }^{2}$ Center for Cellular and Structural Biology, Sun Yat-Sen University, \\ Guangzhou, Guangdong 510006; ${ }^{3}$ Ying Rui, Inc.; ${ }^{4}$ School of Biosciences and Biopharmaceutics, \\ Guangdong Pharmaceutical University, Guangzhou, Guangdong 510009, P.R. China
}

Received February 26, 2017; Accepted March 7, 2018

DOI: $10.3892 / \mathrm{ol} .2018 .8698$

\begin{abstract}
Bispecific antibodies have been actively studied for cancer therapy due to their potent cytotoxicity against tumor cells. A number of bispecific antibody formats have exhibited strong tumor cytotoxicity in vitro and in vivo. However, effective production of bispecific antibodies remains challenging for the majority of bispecific antibody formats. In the present study, a bispecific antibody was designed that links a conventional antigen-binding fragment (Fab) against cluster of differentiation 3 antigen (CD3) to a camel single domain antibody (VHH) against human epidermal growth factor receptor 2 (HER2). This bispecific antibody may be secreted and purified efficiently from Escherichia coli culture medium. The purified bispecific antibody is able to trigger $\mathrm{T}$ cell-mediated HER2-specific cytotoxicity in vitro and in vivo. The data gathered in the present study suggest that this bispecific format may be applied to other tumor antigens to produce bispecific antibodies more efficiently.
\end{abstract}

\section{Introduction}

Human epidermal growth factor receptor 2 (HER2), is overexpressed in $\sim 25 \%$ of patients with metastatic breast carcinoma and a number of other human cancer types, including gastric, lung, ovarian, bladder and kidney carcinomas (1-3). As a therapeutic target for HER2-overexpressing cancer (4), monoclonal antibodies have been developed to target HER2-positive tumors (5-7). For example, trastuzumab and pertuzumab have

Correspondence to: Dr Qing Li or Dr Zhong Wang, School of Pharmaceutical Sciences, Sun Yat-Sen University, 132 Waihuan East Road, Higher Education Megacenter, Guangzhou, Guangdong 510006, P.R. China

E-mail: liqing66@mail.sysu.edu.cn

E-mail: wangzh357@mail.sysu.edu.cn

Key words: human epidermal growth factor receptor 2, cluster of differentiation 3, bispecific antibody, extracellular expression, Escherichia coli already been approved clinically for HER2-positive breast cancer $(8,9)$. Trastuzumab and pertuzumab are able to directly inhibit HER2 activities and induce antibody-dependent cell-mediated cytotoxicity. The two antibodies may increase the survival time when combined with chemotherapy in patients with HER2-overexpressing breast cancer (10-12). However, for the majority of patients with metastatic breast cancer, the tumors eventually resist trastuzumab, and certain patients do not respond to treatment even with HER2 overexpression $(13,14)$.

In order to improve the therapeutic effect of antibodies, a number of approaches have been studied, including antibody conjugate TDM1 (15). Another approach is to directly engage immune cells to attack tumor cells. As T cells serve an important function in the killing of tumor cells (16-20), bispecific antibody that recruits $\mathrm{T}$ cells to kill tumor cells is of interest and has been investigated for cancer therapy (21-23). For example, blinatumomab, a bispecific $\mathrm{T}$ cell engager antibody (BiTE), has been approved for the treatment of B-cell leukemia (23). Numerous bispecific antibodies targeting different tumor biomarkers, including HER2, have also been reported (24-27).

The present study reports on a T-cell engaging bispecific antibody, cluster of differentiation (CD)3-S-Fab, which targets HER2 tumor cells. Distinct from previous studies (28-30), CD3-S-Fab was designed by linking a camel anti-HER2 single-domain antibody (VHH) to the C-terminal of a conventional anti-CD3 antigen-binding fragment (Fab). The CD3-S-Fab may be expressed and purified from Escherichia coli. To improve the purification process, different expression and purification schemes were tested, and it was identified that CD3-S-Fab may be secreted and purified directly from $E$. coli medium with high efficiency. The purified CD3-S-Fab is able to recruit T cells to kill HER2-positive tumor cells specifically. The data gathered in the present study demonstrate that CD3-S-Fab may present a feasible approach to produce bispecific antibodies on a large scale.

\section{Materials and methods}

Plasmids. To make the CD3-S-Fab bispecific antibody, the VH-CH1 and VL-CL of anti-CD3 UCHT1 clone (31) 
were synthesized (Genscript Biotech., Nanjing, China). The VH-CH1 was cloned into the pET26b plasmid (Addgene, Inc., Cambridge, MA, USA) through restriction enzyme cutting site $\mathrm{NcoI}$ and BamHI (Fig. 1A). The VL-CL of anti-CD3 UCHT1 was linked with the single domain anti-HER2 VHH (8), and then cloned into the pET21a (Addgene, Cambridge, MA, USA) through restriction enzyme cutting site $\mathrm{NcoI}$ and $\mathrm{XhoI}$ (Fig. 1A). The pelB signal sequence (5'-ATGAAATACCTGCTGCCG ACCGCTGCTGCTGGTCTGCTGCTCCTCGCTGCCCAG CCGGCGATGGCCATGG-3') was synthesized (Genscript Biotech., Nanjing, China) and added to the N-terminals of the two constructs for periplasmic expression $(32,33)$. A Flag-tag or His-tag (Genscript, Nanjing, Jiangsu, China) was added to the C-terminals for easy detection.

Bispecific antibody expression and purification. In order to purify the CD3-S-Fab protein, E. coli BL21(DE3) competent cells were transformed with the two plasmids encoding VH-CH1 and VL-CL-HER2VHH. Briefly, competent cells and plasmids were mixed and incubated at $42^{\circ} \mathrm{C}$ for $45 \mathrm{sec}$, then cooled on ice for $2 \mathrm{~min}$. After incubating cells for $1 \mathrm{~h}\left(37^{\circ} \mathrm{C}\right)$, cells were spread on lysogeny broth (LB) plates and incubated at $37^{\circ} \mathrm{C}$ for $12 \mathrm{~h}$. For periplasmic expression, the bacteria were cultured in (LB) medium (10 g/l tryptone, $5 \mathrm{~g} / 1$ yeast extract and $10 \mathrm{~g} / 1 \mathrm{NaCl}$; Sangon Biotech; Shanghai, China) with antibiotics $(0.1 \mathrm{~g} / \mathrm{l}$ Ampicillin plus $0.05 \mathrm{~g} / 1 \mathrm{Kanamycin})$ at $37^{\circ} \mathrm{C}$ until the optical density at a wavelength of $600 \mathrm{~nm}\left(\mathrm{OD}_{600}\right)$ (measured by NanoDrop2000; Thermo Fisher Scientific, Inc.). Next, isopropyl $\beta$-D-1-thiogalactopyranoside (IPTG) was added to a final concentration of $0.1 \mathrm{mM}$ to induce protein expression, and cell growth was continued for an additional $20 \mathrm{~h}$ at $16^{\circ} \mathrm{C}$ or $4 \mathrm{~h}$ at $37^{\circ} \mathrm{C}$ using constant rotary incubator (Zhicheng Inc; Shanghai, China) at $180 \mathrm{rpm}$. Periplasmic protein purification was performed as described previously (34). Briefly, cells were harvested with centrifugation at $4,000 \mathrm{x} \mathrm{g}$ for $30 \mathrm{~min}$ at $4^{\circ} \mathrm{C}$ ) and the cell pellet was resuspended in a chilled sucrose solution $(20 \mathrm{mM}$ Tris- $\mathrm{HCl} \mathrm{pH} 8.0 ; 25 \%$ (w/v) sucrose; $1 \mathrm{mM}$ EDTA). Following incubation on ice for 15 min with occasional agitation, the suspension was then centrifuged at $8,500 \mathrm{x} \mathrm{g}$ for $20 \mathrm{~min}$ at $4^{\circ} \mathrm{C}$. The supernatant was collected as the sucrose fraction. The cells were resuspended again and incubated in chilled periplasmic solution $(5 \mathrm{mM} \mathrm{MgCl}$ ) for an additional $30 \mathrm{~min}$. Following centrifugation $\left(20,000 \mathrm{xg}, 4^{\circ} \mathrm{C}\right.$ for $\left.30 \mathrm{~min}\right)$, the supernatant was collected as the periplasmic fraction.

To test the secreted expression, M9 minimal medium (Sangon Biotech Co., Ltd., Shanghai, China) was used as described previously $(32,35)$. Briefly, the bacteria transformed with the two plasmids were cultured in LB medium with antibiotics at $37^{\circ} \mathrm{C}$. The culture was then transferred to M9 minimal medium $\left(12.8 \mathrm{~g} / 1 \mathrm{Na}_{2} \mathrm{HPO}_{4}, 3.0 \mathrm{~g} / 1 \mathrm{KH}_{2} \mathrm{PO}_{4}, 0.5 \mathrm{~g} / \mathrm{l}\right.$ $\mathrm{NaCl}, 2.0 \mathrm{~g} / 1 \mathrm{NH}_{4} \mathrm{Cl}, 20 \mathrm{~g} / 1$ glucose, $0.1 \mathrm{mM} \mathrm{CaCl}{ }_{2}, 1.0 \mathrm{mM}$ $\mathrm{MgSO}_{4}$ and $10 \mu \mathrm{M} \mathrm{FeCl}_{3}$ ), and incubated at $37^{\circ} \mathrm{C}$ and $220 \mathrm{rpm}$ in a rotary shaker. When the cell culture reached an $\mathrm{OD}_{600}$ of 2.7-2.9, IPTG (final concentration, $1 \mathrm{mM}$ ) and Tris- $\mathrm{HCl}$ (final concentration, $180 \mathrm{mM}$ ) were added to induce protein expression and secretion. Following culture for another $24 \mathrm{~h}$ at $16^{\circ} \mathrm{C}$ and $220 \mathrm{rpm}$ in a rotary shaker, the cells were removed by centrifugation $\left(4,000 \times \mathrm{g}, 4^{\circ} \mathrm{C}, 30 \mathrm{~min}\right.$ followed by $20,000 \mathrm{x} \mathrm{g}$, $4^{\circ} \mathrm{C}, 30 \mathrm{~min}$ ) and the supernatant was recovered and processed for purification as follows:

CD3-S-Fab was purified from the combined sucrose and periplasmic fractions or protein containing medium using Ni-NTA agarose (cat. no., NINTA-300; Molecular Cloning Laboratories, San Francisco, CA, USA) via a C-terminal His8-Tag. Purified protein was then further analyzed by SDS-PAGE. Briefly, $10 \mu \mathrm{g}$ per protein sample was separated on $12 \%$ SDS-PAGE gel under reducing conditions by adding 2 uM 2-mercaptoethanol, then the gel was stained by coomassie brilliant blue solution for $1 \mathrm{~h}$ at room temperature (Sigma-Aldrich; Merck KGaA, Darmstadt, Germany). After destaining, the gel with water 3 times for 5 min each, the gel was photographed by ChemiDoc XRS (Bio-Rad Laboratories, Inc., Hercules, CA, USA). The concentration of purified protein was determined by NanoDrop2000 (Thermo Fisher Scientific, Inc.).

Cell lines and animals. All cell lines, namely CHO, SKBR-3 and LS174T (HER2+) cells, and Jurkat T cells, were purchased from the Type Culture Collection of the Chinese Academy of Sciences (Shanghai, China). SKBR-3 cells were cultured in Dulbecco's modified Eagle's medium (Gibco; Thermo Fisher Scientific, Inc., Waltham, MA, USA) with $10 \%$ heat-inactivated fetal bovine serum (HI-FBS; Gibco; Thermo Fisher Scientific, Inc.) and $1 \%$ penicillin/streptomycin. LS174T, CHO and Jurkat cells were cultured in RPMI-1640 medium (Gibco; Thermo Fisher Scientific, Inc.) with $10 \%$ HI-FBS (Gibco; Thermo Fisher Scientific, Inc.) and $1 \%$ penicillin/streptomycin. All cells were incubated at $37^{\circ} \mathrm{C}$ in a humidified incubator with $5 \% \mathrm{CO}_{2}$.

A total of 10 of non-obese diabetic-severe combined immunodeficiency disease (NOD/SCID) mice (female, $\sim 18 \mathrm{~g}, 6$-week-old) were purchased from Beijing Vital River Laboratory Animal Technology, Co, Ltd. (Beijing, China) and housed in the Animal Experiment Center of Sun Yat-Sen University (Guangzhou, China) under sterile and standardized environmental conditions $\left(20-26^{\circ} \mathrm{C}\right.$ room temperature, free access to food and water, $40-70 \%$ relative humidity and $12 \mathrm{~h}$ light-dark cycle). Animal care and experimental procedures were approved by the Institutional Animal Care and Use Committee, Sun Yat-Sen University.

Flow cytometry analysis. Flow cytometry analysis was performed as described previously $(8,29)$. Briefly, aliquots of $1 \times 10^{6}$ cells were collected and mixed in ice-cold PBS with $0.2 \%$ bovine serum albumin (BioTeK China, Beijing, China) in the absence or presence of CD3-S-Fab (final concentration of $50 \mu \mathrm{g} / \mathrm{ml}$ ). The mixture was then incubated on ice for $1 \mathrm{~h}$, followed by washing twice with ice-cold PBS. The cells were then incubated on ice for $1 \mathrm{~h}$ with goat-anti-human immunoglobulin (Ig)G (H+L)-AF488 (1:200, cat. no. A11013; Invitrogen; Thermo Fisher Scientific, Inc.) as the secondary antibody. The cells were also incubated with anti-CD3-FITC antibody (5 $\mu 1$ /test, BioLegend, Inc., San Diego, CA, USA; cat. no. 317306), anti-HER2-PE antibody (5 $\mu 1 /$ test, cat. no. 340552; BD Biosciences, Franklin lakes, NJ, USA) or goat-anti-human IgG (H+L)-AF488 (1:200, cat. no. A11013; Invitrogen; Thermo Fisher Scientific, Inc.) on ice for $1 \mathrm{~h}$. After the cells were washed twice by cold PBS, flow cytometry analysis was performed by 


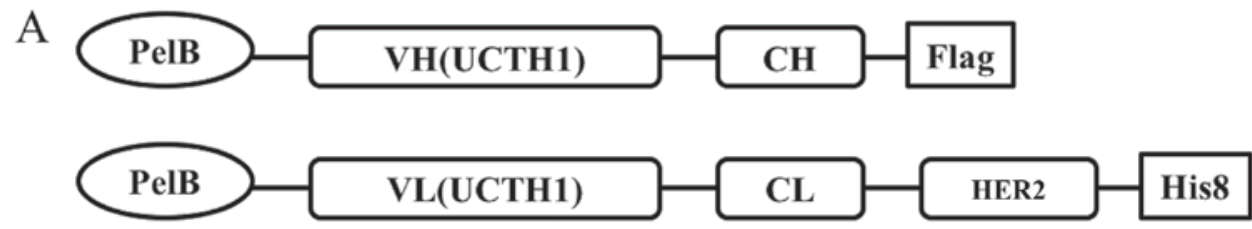

B

C $\quad$ MW (kDa)

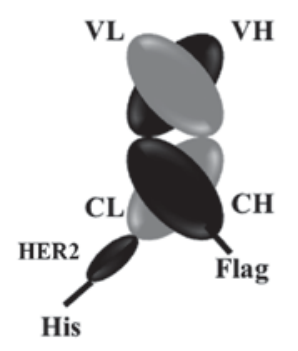

CD3-S-Fab

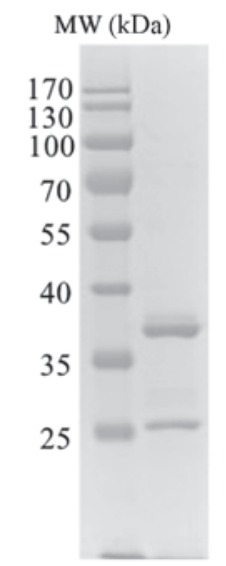

Figure 1. CD3-S-Fab purification from E. coli. (A) The constructs of CD3-S-Fab. Each construct contains a PelB signal sequence, VH-CH1 or VL-CL of humanized anti-CD3 (UTCH1 clone) and an anti-HER2 VHH. To facilitate protein detection and purification, a Flag-tag or a His8-tag was added to the c-terminal of each construct. (B) Formation of CD3-S-Fab after co-expression. (C) Coomassie blue-staining of CD3-S-Fab purified with immobilized Ni-NTA affinity chromatography. HER2, human epidermal growth factor receptor 2; CD, cluster of differentiation.

the Cytomics FC500 Flow Cytometer (Beckman Coulter, Inc., Brea, CA USA).

Isolation of $T$ cells from peripheral blood mononuclear cells (PBMCs). Human PBMCs were retrospectively obtained from healthy donors from the Guangzhou Blood Centre (Guangzhou, China), which provided informed consent (approval no. SYSU-2015-289) using Ficoll-Paque PLUS (cat. no. 17-1440-03; GE Healthcare, Chicago, IL, USA) density centrifugation, as described previously (36). The use of the cells was approved by the Health and Family Planning Commission of Guangdong Province. In brief, $25 \mathrm{ml}$ two-fold diluted peripheral blood from healthy donors was layered on $15 \mathrm{ml}$ Ficoll-Paque PREMIUM and centrifuged at $400 \mathrm{x} \mathrm{g}$ for $30 \mathrm{~min}$ at room temperature. PMBCs were collected and washed three times with PBS. T cells were then enriched from PBMCs using an EasySep ${ }^{\mathrm{TM}}$ Human CD3 Positive Selection kit (Stemcell Technologies, Inc., Vancouver, BC, Canada) as descried previously (37). The isolated T cells were cultured in complete RPMI 1640 with $10 \% \mathrm{FBS}$ and $1 \%$ penicillin/streptomycin at $37^{\circ} \mathrm{C}$ in $5 \%$ $\mathrm{CO}_{2}$ prior to usage.

Cytotoxic assays. Cytotoxicity assays were performed as described previously (29). Briefly, SKBR3, LS174T and CHO cells were used as target cells. T cells without prior stimulation were used as effector cells. A total of 5,000 cells/well of target cells $(100 \mu \mathrm{l})$ was plated in 96-well plates in triplicate. Following a 6-24 $\mathrm{h}$ incubation period, an equal volume of $\mathrm{CD}^{+} \mathrm{T}$ cells $(50,000$ cells/well) or complete RPMI 1640 medium were added to each well. The CD3-S-Fab and Trastuzumab (a gift from Alphamab, Suzhou, China), which is an approved monoclonal antibody to treat HER2 positive patients with breast cancer, ranging from $1.56 \times 10^{2}$ to $1.56 \times 10^{-5} \mathrm{nM}$, were then added. After $72 \mathrm{~h}$ of incubation, cell viability was quantified using cell counting kit (cat. no. CK04; Dojindo Molecular Technologies, Inc., Kumamoto, Japan) according to the manufacturer's protocol. The survival rate (\%) of target cells was calculated using the following formula: [(live target cells (sample)-medium)/(live target cells (control)-medium)] x100.

In vivo efficacy studies. In vivo efficacy studies were performed as described previously, with modifications $(18,38)$. Briefly, HER2-positive SKOV3 cells were harvested and then mixed with freshly isolated human PBMCs. Cell suspensions were injected subcutaneously into the right flank of NOD/SCID mice in a total volume of $0.2 \mathrm{ml} /$ mouse (mixtures of $2 \times 10^{6} \mathrm{SKOV} 3$ cells and $1 \times 10^{7}$ human PBMCs). The mice were grouped into control group (PBS) and treatment group (CD3-S-Fab) randomly, 5 mice per group. The first antibody treatment $(1 \mathrm{mg} / \mathrm{kg}$ ) was at $2 \mathrm{~h}$ post-transplantation. The animals were then treated daily $(1 \mathrm{mg} / \mathrm{kg})$ over the following 7 days. Tumor volume was measured daily. Mice were sacrificed when the tumor volume reached $1,500 \mathrm{~mm}^{3}$. All results are presented as the mean for each group.

Statistical analysis. Statistical analysis was performed using GraphPad Prism 7.0 software (GraphPad Software, Inc., La Jolla, CA, USA). Statistical analysis was performed using Student's t-test, except for the T cell-mediated cytotoxicity assay, in which two-way ANOVA followed by Dunnett's multiple comparisons test was employed. A non-linear regression analysis was used in Fig. 3B-E. $\mathrm{P}<0.05$ was considered a statistically significant difference. Data are presented as the mean \pm standard error of the mean unless otherwise noted. 

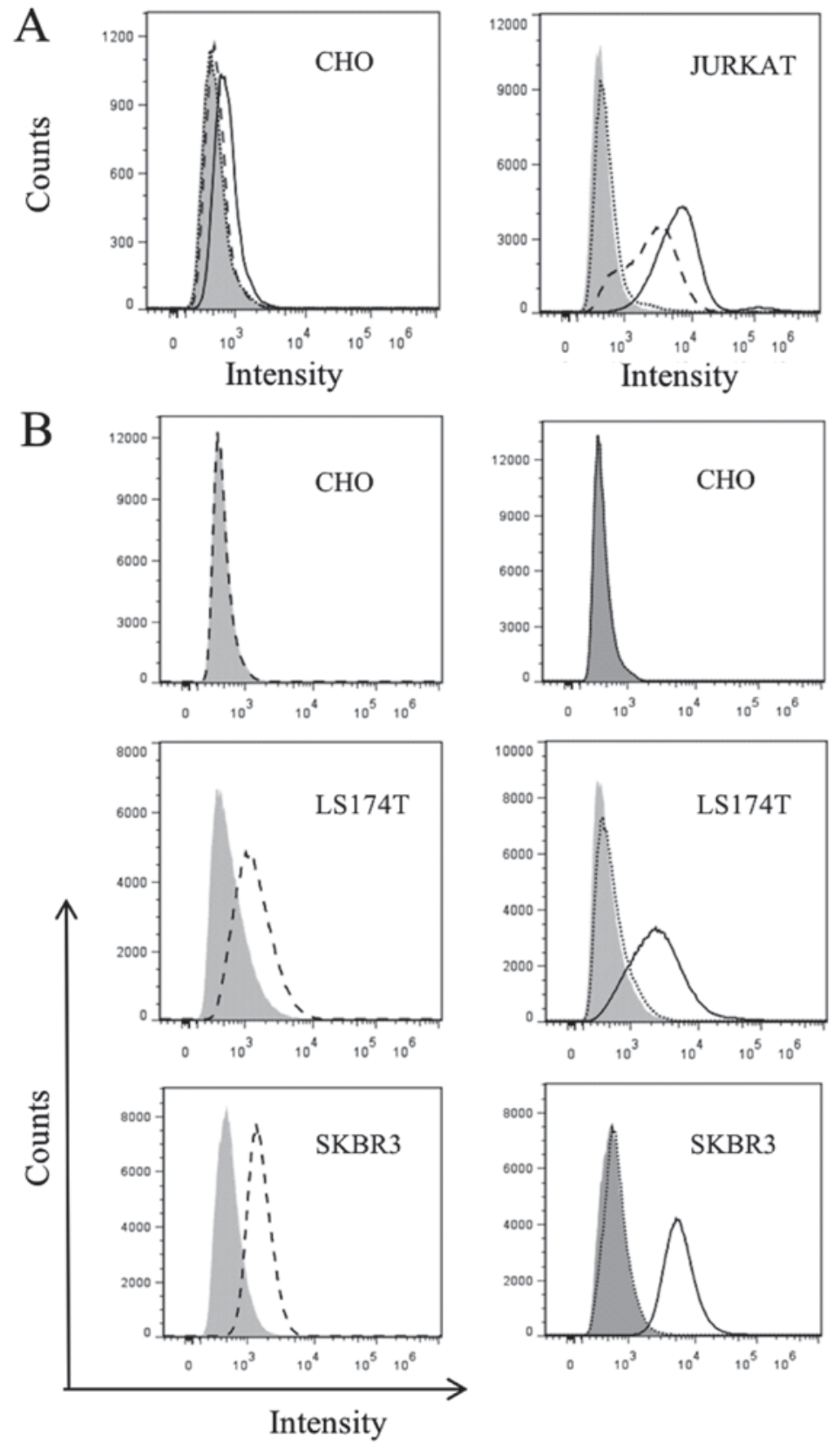

Figure 2. Purified S-Fab can recognize T cells and HER2 positive cells. (A) Flow cytometry analysis with CD3-S-Fab (black line), positive control anti-CD3-FITC (dash line) or staining with only anti-human IgG-AF488 staining (dotted line), on CD3-negative CHO cells (left panel) and CD3-positive Jurkat cells (right panel). (B) Flow cytometry analysis with positive control anti-HER2-PE (A fluorescent protein) (dash line, left panels), CD3-S-Fab (black line, right panels) or staining with only anti-human IgG-AF488 (dotted line), on HER2-negative CHO cells (top panel) and HER2-positive cell lines, LS174T and SKBR3 (bottom panels). HER2, human epidermal growth factor receptor 2; Ig, immunoglobulin; CD, cluster of differentiation.

\section{Results}

CD3-S-Fab may be secreted and purified from E. coli culture medium. CD3-S-Fab was designed by genetically linking an anti-HER2 VHH at the c-terminal of anti-CD3 VL-CL (Fig. 1A). Anti-CD3 VH-CH1 and anti-CD3 VL-CL-VHH were cloned into pET26b and pET21a, respectively. The pelB signal peptide was added to the N-terminal of the two constructs for periplasmic expression and secretion in E. coli. The CD3-S-Fab was formed via the heterodimerization of VH-CH1/VL-CL-VHH (Fig. 1A).

Periplasmic purification was tested first by adjusting the IPTG concentrations and culture temperature. The optimal expression with improved solubility of CD3-S-Fab was achieved by lowering the induction temperature $(0.1 \mathrm{mM}$ IPTG, $16^{\circ} \mathrm{C}$ induction for $24 \mathrm{~h}$; data not shown). However, the yield of CD3-S-Fab remained low with a yield of $\sim 0.4 \mathrm{mg}$ per 6 liters LB medium following Ni-NTA affinity purification.

To increase the yield of CD3-S-Fab, extracellular expression of CD3-S-Fab was tested $(39,40)$. Compared with the periplasmic expression, the yield of CD3-S-Fab recovered from the M9 medium was $0.6 \mathrm{mg}$ per $200 \mathrm{ml}$ medium. The secreted CD3-S-Fab was also able to be purified by Ni-NTA-agarose affinity chromatography as heterodimers (Fig. 1C). Thus, CD3-S-Fab may be secreted and purified from E. coli culture medium. 

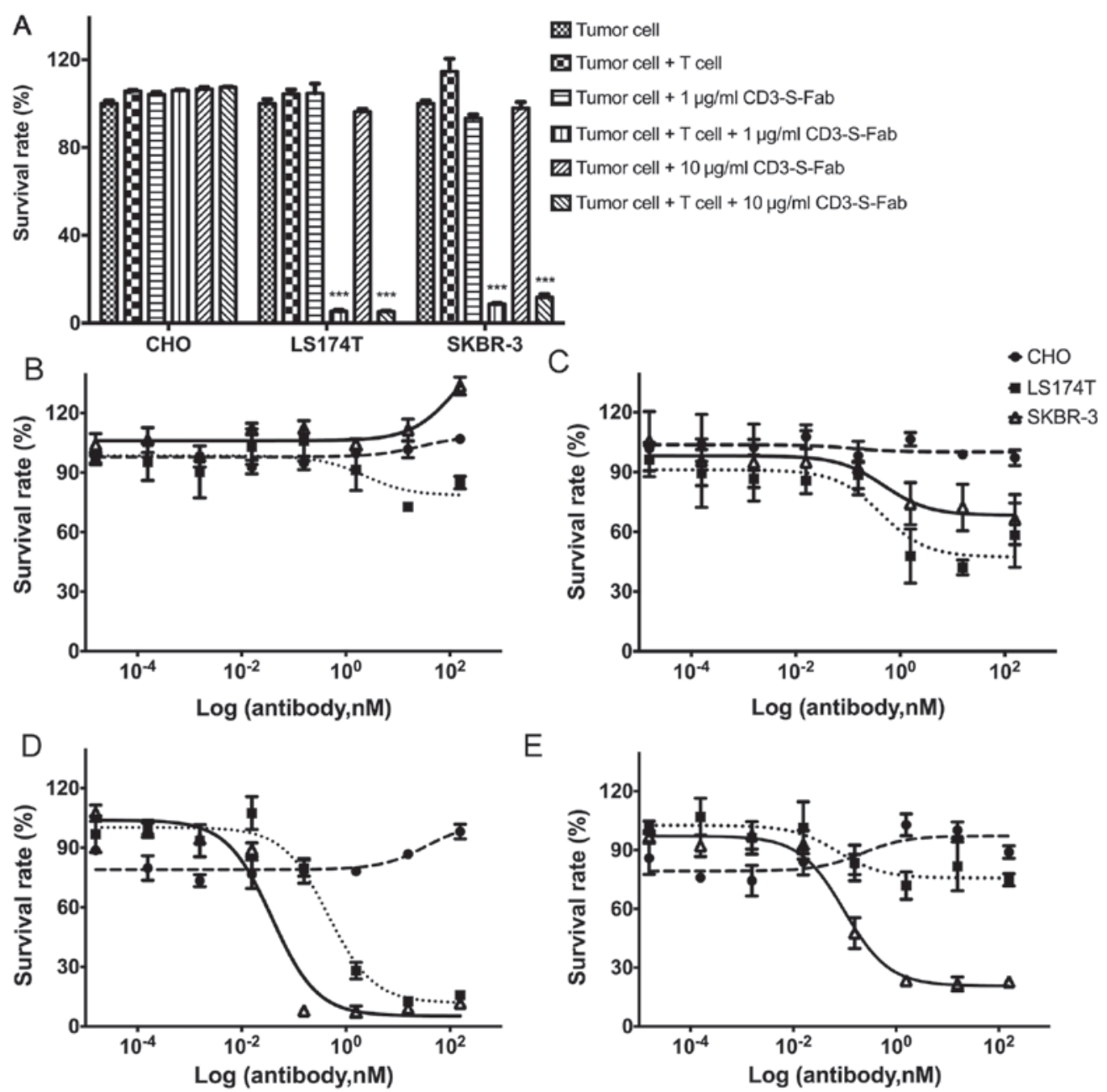

Figure 3. CD3-S-Fab kills tumor cells in a T cell-dependent and tumor antigen-dependent manner. (A) Tumor cells (5,000 cells/well) alone or with T cells (50,000 cells/well) were incubated together for $72 \mathrm{~h}$ in the presence of the indicated concentrations of CD3-S-Fab ( 1 or $10 \mu \mathrm{g} / \mathrm{ml})$. Cytotoxic activity was measured as described in the Materials and methods section. Data are presented as the mean $\pm \operatorname{SEM}\left(n=3,{ }^{* * *} \mathrm{P}<0.001\right.$ vs. tumor cells $+\mathrm{T}$ cells, two-way analysis of variance followed by Dunnett's multiple comparisons test). Dose response measurement of CD3-S-Fab with CHO (circle), LS174T (square) and SKBR-3 (triangle) cells. Dose-response curves were assessed using a non-linear regression, log (inhibitor) vs. response using GraphPad Prism software; (B) in the absence of T cells with different concentrations of CD3-S-Fab, (C) in the presence of T cells with different concentrations of CD3-S-Fab, (D) in the absence of PBMCs with different concentrations of Trastuzumab, and (E) in the presence of PBMCs with different concentrations of Trastuzumab. All measurements were normalized against tumor cells only; data points in the figure represent the mean of three samples and error bars represent the SEM. SEM, standard error of the mean; PBMC, peripheral blood mononuclear cell; CD, cluster of differentiation.

CD3-S-Fab binds CD3- and HER2-positive cells. In order to confirm whether CD3-S-Fab maintains the ability to bind CD3-positive T cells, flow cytometry analysis was conducted using CD3-positive Jurkat cells and CD3-negative CHO cells. CD3-S-Fab was not able to bind $\mathrm{CHO}$ cells based on flow cytometry analysis (Fig. 2A), but was able to bind Jurkat cells (Fig. 2B), suggesting that CD3-S-Fab may bind human $\mathrm{T}$ cells.

To confirm the binding of CD3-S-Fab to HER2-positive tumor cells, HER2-positive cell lines, SKBR3 and LS174T, and the HER2-negative cell line CHO, were used for flow cytometry analysis. Flow cytometry analysis revealed that CD3-S-Fab did not bind to CHO cells, but that it did bind to SKBR3 and LS174T cells (Fig. 2B, right panels). These data suggest that CD3-S-Fab is able to specifically bind to HER2-positive cells.

CD3-S-Fab has T-cell-mediated cytotoxicity against HER2-positive cells. In order to evaluate whether CD3-S-Fab

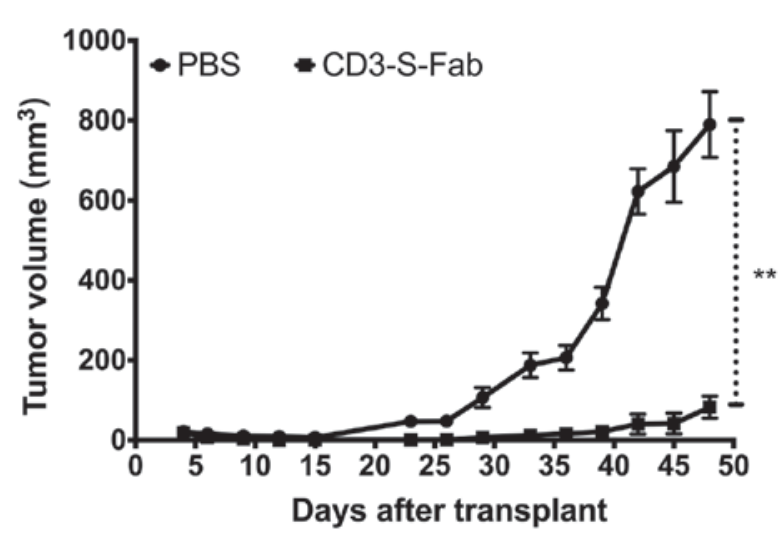

Figure 4. CD3-S-Fab inhibits tumor growth in vivo. Non-obese diabetic-severe combined immunodeficiency disease mice ( $n=5 /$ group) were engrafted subcutaneously with SKOV3 cells and human PBMCs. The mice were then treated with PBS (solid square, PBMC transplant) or CD3-S-Fab (solid circle, PBMC transplant). The data represent the mean tumor volume of 5 mice. Error bars represent standard error of the mean $\left({ }^{* *} \mathrm{P}<0.01\right.$ vs. PBS group, paired t-test). PBMC, peripheral blood mononuclear cell; $\mathrm{CD}$, cluster of differentiation. 
is able to mediate HER2 tumor cell killing, HER2-positive and HER2-negative cell lines were used. CD3-S-Fab did not lead to cytotoxicity in the HER2-negative cell line CHO (Fig. 3A and B). For the HER2-positive cell lines LS174T and SKBR3, T cells alone or CD3-S-Fab alone have no effects on cell viability (Fig. 3B). However, CD3-S-Fab induced potent cytotoxicity when the LS174T and SKBR3 cells were incubated with CD3-S-Fab and T cells (Fig. 3A). Cell number and morphology observed under microscopy also confirmed the specific killing in the presence of CD3-S-Fab and T cells.

To further evaluate the cytotoxic activity of CD3-S-Fab on tumor cells, the dose responses of different cell lines were measured. No cell killing was observed for CD3-S-Fab in the absence of T cells (Fig. 3B). With T cells present, CD3-S-Fab exhibited active cell killing in HER2-positive LS174T cells and SKBR3 cells (Fig. 3C). This is distinct from Trastuzumab, which demonstrated partial inhibition in the absence of PBMCs (Fig. 3D), and higher cell killing in the presence of PBMCs (Fig. 3E). These results suggest that CD3-S-Fab exhibits potent cytotoxic activity against HER2-positive tumor cells in the presence of $\mathrm{T}$ cells.

CD3-S-Fab inhibits tumor growth in vivo. To analyze the in vivo antitumor effect of CD3-S-Fab, SKOV3 cells were mixed with freshly isolated human PBMCs and engrafted subcutaneously into NOD/SCID mice. The mice were then treated with either PBS or CD3-S-Fab. Compared with animals only treated with PBMCs, significant tumor growth inhibition was observed in mice treated with CD3-S-Fab (Fig. 4). Minimal tumor growth was observed in mice treated with CD3-S-Fab, even 5 weeks after treatment ended. These data demonstrated that CD3-S-Fab was able to inhibit HER2-positive tumor growth in xenograft mice.

\section{Discussion}

Cancer immunotherapy has demonstrated lasting clinical benefits in patients with cancer (41). Besides checkpoint antibodies, a variety of approaches have been actively studied as cancer immunotherapies. Among them, bispecific antibodies have demonstrated some promise. For example, blinatumomab, a BiTE, has already been approved for the treatment of B-cell leukemia, with excellent efficacy $(18-21,23,42)$.

HER2 is one of the most studied oncogenes. Antibodies or small molecule inhibitors have exhibited clinical efficacy by inhibiting HER2 activity. Besides functioning as an oncogene, HER 2 also presents as an excellent tumor antigen, as it is overexpressed in numerous tumors and is rarely expressed in normal tissues $(4,8,43-46)$. Vaccines, bispecific antibodies and other approaches have been studied to further improve the clinical outcomes of current HER2 therapeutics.

Different formats of anti-HER2 bispecific antibodies have been studied previously $(15,25,28,45,47,48)$, including recruitment of $\mathrm{T}$ cells in various bispecific antibody formats $(17,19,49,50)$. However, those bispecific antibodies present a number of challenges, including a mixed population during purification, a low yield of production, a tendency to aggregate and a short half-life. Previously, it was demonstrated that an S-Fab bispecific antibody against carcinoembryonic antigens demonstrated several advantages, including excellent efficacy, and reasonable expression and solubility in E. coli (29). In the present study, CD3-S-Fab, the bispecific antibody targeting HER2, is described. The purified bispecific antibody CD3-S-Fab can be used for the redirection of $\mathrm{T}$ cells toward HER2-positive tumor cells, and was demonstrated to be efficient in vitro and in vivo at killing HER2-positive cancer cells.

Although CD3-S-Fab may be produced in E. coli, the yield of CD3-S-Fab was very low based on purification from periplasmic fractions. Recombinant antibodies are commonly produced by eukaryotic cells or periplasmic expression of gram-negative bacteria $(8,18,21,35,50,51)$. Due to the easy culture and low cost, E. coli has been widely used as an expression host for recombinant proteins. However, the high yield of correctly folded proteins is a frequent problem, and the purification process of the recombinant proteins is also complicated. In the present study, the defined M9 medium was used to facilitate the secretion of CD3-S-Fab complex into the medium, and then the recombinant antibody was purified directly from this medium. This purification schedule greatly increased the yields of CD3-S-Fab.

Compared with the intracellular expression of recombinant proteins, extracellular expression exhibits several advantages, including the following: i) It is efficient at obtaining correctly folded proteins; ii) the secreted proteins are less likely to be degraded by various proteases in the periplasm; iii) secretion reduces the cellular burden for cell growth when a large amount of recombinant protein is produced; and iv) The process of purification is easier due to the elimination of cellular component contamination $(33,40,52,53)$.

In summary, the novel bispecific antibody CD3-S-Fab can be used for the redirection of T cells toward HER2-positive tumor cells and is efficient at killing HER2-positive cancer cells in vitro and in vivo. The easy purification and high yield of CD3-S-Fab suggests this format may be applied to other bispecific antibodies.

\section{Acknowledgements}

The authors would like to thank Dr. Jiang Li at the Sun Yat-Sen University School of Medicine and Dr. Wei Xie at the Sun Yat-Sen University School of Life Sciences, for their technical support.

\section{Funding}

This study was financially supported by the R\&D Plan of Guangdong Province (China) (grant no. 20160503).

\section{Availability of data and materials}

The datasets used and/or analyzed during the current study are available from the corresponding author on reasonable request.

\section{Authors' contributions}

LL, LLi, CZ, JL, JLiu, RS, and BD, performed the experiments. LL, QL and ZW designed the experiments, and wrote the manuscript. 


\section{Ethics approval and consent to participate}

The use of animals was approved by the Institutional Animal Care and Use Committee, Sun Yat-Sen University (Guangzhou, China). (Approve No. IACUC-DD-18-02-01). The use of human blood was approved by Health and Family Planning Commission of Guangdong Province (approval no. SYSU 2015-289).

\section{Consent for publication}

The PBMCs were from provided from Health and Family Planning Commission of Guangdong Province (SYSU 2015-289) with consent from healthy donors.

\section{Competing interests}

The authors declare that they have no competing interests.

\section{References}

1. Slamon DJ, Clark GM, Wong SG, Levin WJ, Ullrich A and McGuire WL: Human breast cancer: Correlation of relapse and survival with amplification of the HER-2/neu oncogene. Science 235: 177-182, 1987.

2. Press MF, Pike MC, Hung G, Zhou JY, Ma Y, George J, Dietz-Band J, James W, Slamon DJ, Batsakis JG, et al: Amplification and overexpression of HER-2/neu in carcinomas of the salivary gland: Correlation with poor prognosis. Cancer Res 54: 5675-5682, 1994.

3. Daniele L and Sapino A: Anti-HER2 treatment and breast cancer: State of the art, recent patents, and new strategies. Recent Pat Anticancer Drug Discov 4: 9-18, 2009.

4. Yarden Y: Biology of HER 2 and its importance in breast cancer. Oncology 61 (Suppl 2): S1-S13, 2001.

5. Ben-Kasus T, Schechter B, Lavi S, Yarden Y and Sela M: Persistent elimination of ErbB-2/HER2-overexpressing tumors using combinations of monoclonal antibodies: Relevance of receptor endocytosis. Proc Natl Acad Sci USA 106: 3294-3299, 2009.

6. KelerT, Graziano RF, Mandal A, Wallace PK, Fisher J, Guyre PM, Fanger MW and Deo YM: Bispecific antibody-dependent cellular cytotoxicity of HER2/neu-overexpressing tumor cells by Fc gamma receptor type I-expressing effector cells. Cancer Res 57: 4008-4014, 1997.

7. Vasconcellos FA, Aleixo PB, Stone SC, Conceicao FR, Dellagostin OA and Aleixo JA: Generation and characterization of new HER2 monoclonal antibodies. Acta Histochem 115: 240-244, 2013.

8. Vaneycken I, Devoogdt N, Van Gassen N, Vincke C, Xavier C, Wernery U, Muyldermans S, Lahoutte $\mathrm{T}$ and Caveliers $\mathrm{V}$ : Preclinical screening of anti-HER2 nanobodies for molecular imaging of breast cancer. FASEB J 25: 2433-2446, 2011.

9. Hicks DG and Kulkarni S: HER2+ breast cancer: Review of biologic relevance and optimal use of diagnostic tools. Am J Clin Pathol 129: 263-273, 2008.

10. Ranson M and Sliwkowski MX: Perspectives on anti-HER monoclonal antibodies. Oncology 63 (Suppl 1): S17-S24, 2002.

11. Hudis CA: Trastuzumab-mechanism of action and use in clinical practice. N Engl J Med 357: 39-51, 2007.

12. Spector NL and Blackwell KL: Understanding the mechanisms behind trastuzumab therapy for human epidermal growth factor receptor 2-positive breast cancer. J Clin Oncol 27: 5838-5847, 2009.

13. Valabrega G, Montemurro F and Aglietta M: Trastuzumab: Mechanism of action, resistance and future perspectives in HER2-overexpressing breast cancer. Ann Oncol 18: 977-984, 2007.

14. Junttila TT, Parsons K, Olsson C, Lu Y, Xin Y, Theriault J, Crocker L, Pabonan O, Baginski T, Meng G, et al: Superior in vivo efficacy of afucosylated trastuzumab in the treatment of HER2-amplified breast cancer. Cancer Res 70: 4481-4489, 2010.

15. Arteaga CL, Sliwkowski MX, Osborne CK, Perez EA, Puglisi F and Gianni L: Treatment of HER2-positive breast cancer: Current status and future perspectives. Nat Rev Clin Oncol 9: 16-32, 2011.
16. Benchetrit F, Gazagne A, Adotevi O, Haicheur N, Godard B, Badoual C, Fridman WH and Tartour E: Cytotoxic T lymphocytes: Role in immunosurveillance and in immunotherapy. Bull Cancer 90: 677-685, 2003.

17. Nagorsen D, Bargou R, Ruttinger D, Kufer P, Baeuerle PA and Zugmaier G: Immunotherapy of lymphoma and leukemia with T-cell engaging BiTE antibody blinatumomab. Leuk Lymphoma 50: 886-891, 2009.

18. Junttila TT, Li J, Johnston J, Hristopoulos M, Clark R, Ellerman D, Wang BE, Li Y, Mathieu M, Li G, et al: Antitumor efficacy of a bispecific antibody that targets HER2 and activates T cells. Cancer Res 74: 5561-5571, 2014.

19. Baeuerle PA and Reinhardt C: Bispecific T-cell engaging antibodies for cancer therapy. Cancer Res 69: 4941-4944, 2009.

20. Schlereth B, Fichtner I, Lorenczewski G, Kleindienst P, Brischwein K, da Silva A, Kufer P, Lutterbuese R, Junghahn I, Kasimir-Bauer S, et al: Eradication of tumors from a human colon cancer cell line and from ovarian cancer metastases in immunodeficient mice by a single-chain Ep-CAM-/CD3-bispecific antibody construct. Cancer Res 65: 2882-2889, 2005.

21. Taki S, Kamada H, Inoue M, Nagano K, Mukai Y, Higashisaka K, Yoshioka Y, Tsutsumi Y and Tsunoda S: A novel bispecific antibody against human CD3 and ephrin receptor A10 for breast cancer therapy. PLoS One 10: e0144712, 2015.

22. Dreier T, Lorenczewski G, Brandl C, Hoffmann P, Syring U, Hanakam F, Kufer P, Riethmuller G, Bargou R and Baeuerle PA: Extremely potent, rapid and costimulation-independent cytotoxic T-cell response against lymphoma cells catalyzed by a single-chain bispecific antibody. Int J Cancer 100: 690-697, 2002.

23. Oak E and Bartlett NL: Blinatumomab for the treatment of B-cell lymphoma. Expert Opin Investig Drugs 24: 715-724, 2015.

24. Haense N, Atmaca A, Pauligk C, Steinmetz K, Marmé F, Haag GM, Rieger M, Ottmann OG, Ruf P, Lindhofer H and Al-Batran SE: A phase I trial of the trifunctional anti HER $2 x$ anti CD3 antibody ertumaxomab in patients with advanced solid tumors. BMC Cancer 16: 420, 2016.

25. Vaishampayan U, Thakur A, Rathore R, Kouttab N and Lum LG: Phase I study of Anti-CD3 x Anti-HER2 bispecific antibody in metastatic castrate resistant prostate cancer patients. Prostate Cancer 2015: 285193, 2015.

26. Cao Y, Axup JY, Ma JS, Wang RE, Choi S, Tardif V, Lim RK, Pugh HM, Lawson BR, Welzel G, et al: Multiformat T-cell-engaging bispecific antibodies targeting human breast cancers. Angew Chem Int Ed Engl 54: 7022-7027, 2015.

27. Zhou Y, Gou LT, Guo ZH, Liu HR, Wang JM, Zhou SX, Yang JL and Li XA: Fully human HER2/cluster of differentiation 3 bispecific antibody triggers potent and specific cytotoxicity of T lymphocytes against breast cancer. Mol Med Rep 12: 147-154, 2015.

28. Li A, Xing J, Li L, Zhou C, Dong B, He P, Li Q and Wang Z: A single-domain antibody-linked $\mathrm{Fab}$ bispecific antibody HER2-S-Fab has potent cytotoxicity against HER2-expressing tumor cells. AMB Express 6: 32, 2016.

29. Li L, He P, Zhou C, Jing L, Dong B, Chen S, Zhang N, Liu Y, Miao J, Wang Z and Li Q: A novel bispecific antibody, S-Fab, induces potent cancer cell killing. J Immunother 38: 350-356, 2015.

30. Vincke C, Loris R, Saerens D, Martinez-Rodriguez S, Muyldermans S and Conrath K: General strategy to humanize a camelid single-domain antibody and identification of a universal humanized nanobody scaffold. J Biol Chem 284: 3273-3284, 2009.

31. Shalaby MR, Shepard HM, Presta L, Rodrigues ML, Beverley PC, Feldmann $\mathrm{M}$ and Carter P: Development of humanized bispecific antibodies reactive with cytotoxic lymphocytes and tumor cells overexpressing the HER2 protooncogene. J Exp Med 175: 217-225, 1992.

32. Freiherr von Roman M,Koller A, von Rüden D and Berensmeier S: Improved extracellular expression and purification of recombinant Staphylococcus aureus protein A. Protein Expr Purif 93: 87-92, 2014.

33. Yoon SH, Kim SK and Kim JF: Secretory production of recombinant proteins in Escherichia coli. Recent Pat Biotechnol 4: 23-29, 2010.

34. Kwong KY and Rader C: E. coli expression and purification of Fab antibody fragments. Curr Protoc Protein Sci Chapter 6: Unit 6.10, 2009.

35. Skrlj N, Serbec VC and Dolinar M: Single-chain Fv antibody fragments retain binding properties of the monoclonal antibody raised against peptide $\mathrm{P} 1$ of the human prion protein. Appl Biochem Biotechnol 160: 1808-1821, 2010. 
36. So EC, Sallin MA, Zhang X, Chan SL, Sahni L, Schulze DH, Davila E, Strome SE and Jain A: A high throughput method for enrichment of natural killer cells and lymphocytes and assessment of in vitro cytotoxicity. J Immunol Methods 394: 40-48, 2013.

37. Busch R, Cesar D, Higuera-Alhino D, Gee T, Hellerstein MK and McCune JM: Isolation of peripheral blood CD4(+) T cells using RosetteSep and MACS for studies of DNA turnover by deuterium labeling. J Immunol Methods 286: 97-109, 2004.

38. Rozan C, Cornillon A, Petiard C, Chartier M, Behar G, Boix C, Kerfelec B, Robert B, Pèlegrin A, Chames P, et al: Single-domain antibody-based and linker-free bispecific antibodies targeting $\mathrm{Fc} \gamma \mathrm{RIII}$ induce potent antitumor activity without recruiting regulatory T cells. Mol Cancer Ther 12: 1481-1491, 2013.

39. Choi JH and Lee SY: Secretory and extracellular production of recombinant proteins using Escherichia coli. Appl Microbiol Biotechnol 64: 625-635, 2004.

40. Fu XY: Extracellular accumulation of recombinant protein by Escherichia coli in a defined medium. Appl Microbiol Biotechnol 88: 75-86, 2010

41. Scott AM, Wolchok JD and Old LJ: Antibody therapy of cancer. Nat Rev Cancer 12: 278-287, 2012.

42. Osada T, Patel SP, Hammond SA, Osada K, Morse MA and Lyerly HK: CEA/CD3-bispecific T cell-engaging (BiTE) antibody-mediated $\mathrm{T}$ lymphocyte cytotoxicity maximized by inhibition of both PD1 and PD-L1. Cancer Immunol Immunother 64: 677-688, 2015.

43. Karagiannis P, Singer J, Hunt J, Gan SK, Rudman SM, Mechtcheriakova D, Knittelfelder R, Daniels TR, Hobson PS, Beavil AJ, et al: Characterisation of an engineered trastuzumab IgE antibody and effector cell mechanisms targeting HER2/neu-positive tumour cells. Cancer Immunol Immunother 58: 915-930, 2009.

44. Lambertini M, Ponde NF, Solinas C and de Azambuja E: Adjuvant trastuzumab: A 10-year overview of its benefit. Expert Rev Anticancer Ther 17: 61-74, 2017.
45. Xin Y, Guo WW, Huang Q, Zhang P, Zhang LZ, Jiang G and Tian Y: Effects of lapatinib or trastuzumab, alone and in combination, in human epidermal growth factor receptor 2-positive breast cancer: A meta-analysis of randomized controlled trials. Cancer Med 5: 3454-3463, 2016.

46. Malenfant SJ, Eckmann KR and Barnett CM: Pertuzumab: A new targeted therapy for HER2-positive metastatic breast cancer. Pharmacotherapy 34: 60-71,2014.

47. Zazo S, Gonzalez-Alonso P, Martin-Aparicio E, Chamizo C, Cristóbal I, Arpí O, Rovira A, Albanell J, Eroles P, Lluch A, et al: Generation, characterization, and maintenance of trastuzumab-resistant HER 2+ breast cancer cell lines. Am J Cancer Res 6: 2661-2678, 2016.

48. James ND, Atherton PJ, Jones J, Howie AJ, Tchekmedyian S and Curnow RT: A phase II study of the bispecific antibody MDX-H210 (anti-HER2 x CD64) with GM-CSF in HER2+ advanced prostate cancer. Br J Cancer 85: 152-156, 2001.

49. Zhu Z and Carter P: Identification of heavy chain residues in a humanized anti-CD3 antibody important for efficient antigen binding and T cell activation. J Immunol 155: 1903-1910, 1995.

50. Loffler A, Kufer P, Lutterbüse R, Zettl F, Daniel PT, Schwenkenbecher JM, Riethmuller G, Dörken B and Bargou RC: A recombinant bispecific single-chain antibody, CD19 x CD3, induces rapid and high lymphoma-directed cytotoxicity by unstimulated T lymphocytes. Blood 95: 2098-2103, 2000.

51. Qasemi M, Behdani M, Shokrgozar MA, Molla-Kazemiha V, Mohseni-Kuchesfahani $\mathrm{H}$ and Habibi-Anbouhi M: Construction and expression of an anti-VEGFR2 Nanobody-Fc fusionbody in NS0 host cell. Protein Expr Purif 123: 19-25, 2016.

52. Mergulhao FJ, Summers DK and Monteiro GA: Recombinant protein secretion in Escherichia coli. Biotechnol Adv 23: 177-202, 2005.

53. Khushoo A, Pal Y, Singh BN and Mukherjee KJ: Extracellular expression and single step purification of recombinant Escherichia coli L-asparaginase II. Protein Expr Purif 38: 29-36, 2004. 